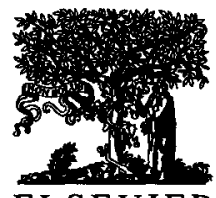

ELSEVIER

\title{
Uncoiling a polymer molecule in a strong extensional flow is
}

\author{
E.J. Hinch \\ Department of Applied Mathematics and Theoretical Physics, University of Cambridge, Silver St., \\ Cambridge CB3 9EW, UK
}

Received 13 April 1994

\begin{abstract}
It has been understood for many years that a polymer molecule will uncoil in a coil-to-stretch transition when it is placed in an extensional flow with a strain rate exceeding the slowest molecular relaxation rate. This paper looks at the effort which must be exerted to achieve this uncoiling, in other words the transient stress in a dilute solution of such uncoiling polymer molecules. Numerical simulations have been performed of an isolated lincar chain of inextensible links which are frecly hinged, the chain being placed in an axisymmetric extension flow. Hydrodynamic interactions between the many beads are not included.

During the uncoiling, the stress is found to be mainly dissipative rather than elastic, i.e. the stress is proportional to the instantaneous strain rate rather than being independent of it. A rapid build up of this viscous stress with the total strain is shown to come from the growth of segments of fully stretched chain. The evolution of these segments, the growth in their size along with the reduction of their number, is examined with a simplified 'kinks dynamics' model.

The above rheological behaviour in transient strong extensional flows is not described by the standard constitutive relations which have been used in the past for dilute polymer solutions, e.g. the Oldroyd-B fluid and FENE dumbbell models. A suitable modification is suggested, which gives large strain-dependent viscous stresses.
\end{abstract}

Keywords: Extensional flow; Kinks dynamics; Polymer uncoiling

"Dedicated to Professor Ken Walters FRS on the occasion of his 60th birthday. 


\section{Introduction}

As at last it has become possible to compute reliably some visco-elastic flows for some simple constitutive relations, there seem to be some discrepancies between the computed flows and the experimental observations. The interesting flows have a large extensional component.

In flow through a contraction, the pressure drop for the Oldroyd-B fluid (Debbaut et al. [1]) and for quite short FENE dumbbells (Keiller [2]) is predicted to be less than that for a Newtonian fluid, whereas experimentally it is observed to be greater. The size of the upstream vortex is also predicted to be smaller than that observed (Boger et al. [3]).

In trying to fit computations to experiments, Rallison and Hinch [4] for the four-roll mill and Chilcott and Rallison [5] for the flow past a sphere found that they needed unexpectedly short FENE chains in order to develop sufficient stress. Similarly, an analysis of the pressure drop across an orifice by Cartolos and Piau [6] in terms of polymers deforming to a rigid finite limit seems to require a finite limit much shorter than of the fully extended chains.

Experiments by Ambari [7] with the same dilute solution of 'Polyox' in different flow geometries could not be understood in terms of the simple dumbbell model, as discussed by Rallison and Hinch [4].

The interesting flows are extensional in nature, strong in terms of a large Weisenberg number, and transient as seen by a fluid particle. Thus this paper will examine the response of an isolated polymer molecule, initially unstressed and coiled, suddenly placed in a strong axisymmetric extensional flow.

There are many simple models of the deformation of a polymer along with the stress that it consequentially exerts. The simplest bead-and-spring dumbbell model is equivalent to the Oldroyd-B fluid, and was derived from considering small strains of the coil. Its unphysical behaviour of infinite and even negative steady extensional viscosities can be rectified with the FENE modifications for large strains. Other modifications include a non-linear friction and a rotation of the beads, and a linear chain of $N$ connected beads and springs (see, for example, Rallison and Hinch [4]). All these models, however, have an elastic polymer stress over and above the Newtonian solvent viscous stress, so that if the flow was instantaneously switched off the polymer stress would not change in that instant. We will see that the numerical simulations of a linear chain do not behave like this in strong flows, but have a mainly viscous stress, i.e. one proportional to the instantaneous strain rate. Thus, in converging flows where the strain rate increases towards the orifice, the stress can increase more rapidly than the strain, which might help explain some of the puzzling experiments.

The need for larger stresses was recognized by James and Sarringer [8] in their experiment which measured the pressure drop in a conical channel. To provide an explanation of these large stresses, King and James [9] suggested that the partially stretched chain might 'freeze', perhaps due to knots or self-entanglements, with the frozen chain behaving rigidly and thence producing the required large dissipative viscous stresses. As a refinement to this model which permitted the chain to 
continue to uncoil whilst producing rigid-like stresses, Ryskin [10] proposed his 'yo-yo' model in which a fully stretched central part of the chain connects the two uncoiling ends. This behaviour was not seen in some early computer simulations by Rallison and Hinch [4], who suggested that the slow unfolding of 'back-loops' was responsible for the rigid behaviour. Larson [11] has recently presented a 'kinks dynamics' model of this unfolding. A further examination of the kinks dynamics will be given in Section 3 .

\section{Computer simulations}

\subsection{Governing equations and methods}

A linear chain is considered to consist of $N$ equal rigid inextensible links (or bonds) which are freely hinged. Note that the links are rigid rather than elastic springs as in the Rouse-Zimm models. Typically $N$ was taken to be 100 , but some simulations have also been performed with $N=50,200,400$ and 800 . The position of each hinge is represented by $\boldsymbol{x}_{i}$, with $i=1, \ldots, N$.

The initial random configuration was generated by choosing the three Cartesian components of the displacment of each link, $\boldsymbol{b}_{i}=\boldsymbol{x}_{i+1}-\boldsymbol{x}_{i}$, uniformly randomly on $[-b, b]$ and then rescaling the vector length to $b$. This process produces a chain with a random configuration, with some small preference for bonds along the diagonals of the co-ordinate axes. flow:

Initially, at $t=0$, the random chain is placed in the axisymmetric cxtensional

$$
\boldsymbol{u}(\boldsymbol{x})=\left(\dot{E} x,-\frac{1}{2} \dot{E} y,-\frac{1}{2} \dot{E} z\right)
$$

with strain rate $\dot{E}>0$. Hydrodynamic beads are allocated to each hinge, on which drag forces are exerted which are proportional to the slip between the imposed extensional flow and the velocity of the bead,

$$
F_{i}=6 \pi \mu a\left(u\left(x_{i}\right)-\dot{x}_{i}\right),
$$

where $\mu$ is the solvent viscosity and $a$ is the hydrodynamic radius of the bead, taken to be constant. No hydrodynamic interactions between the beads are considered.

The chain is also given a Brownian motion by applying during each time-step a random force to each bead. The three Cartesian components of each random bead force $\boldsymbol{B}_{l}$ are selected independently and uniformly randomly on $[-\mathrm{B}, \mathrm{B}]$, with

$$
B-\sqrt{6 k T 6 \pi \mu a / \Delta t}
$$

where $k T$ is the Boltzmann temperature and $\Delta t$ is the size of the time-step. If the beads were not connected on a chain, this magnitude of the random force would produce a displacement in one time-step with a variance of $6 D \Delta t$, where $D=k T / 6 \pi \mu a$ is the diffusivity of the Brownian motion of an isolated bead. This simple prescription of Brownian motion, which was employed in the simulations, is in fact not quite correct when the rigid constraints are applied, as discussed by Grassia et al. [12]. 
To stop the links changing length, a tension $T_{l}$, must be exerted in the link between the $(i+1)$ th and $i$ th beads exerting forces $\pm T_{t} \hat{\boldsymbol{b}}_{t}$ on those beads where $\hat{\boldsymbol{b}}_{t}=\boldsymbol{b}_{t} / \boldsymbol{b}$. It is convenient to define tensions in the non-existent end links as vanishing, i.e. $T_{N}=0=T_{0}^{\prime}$.

Solving the force balance of the $i$ th bead (neglecting inertia) yields the evolution equation for the $i$ th bead:

$$
\dot{\boldsymbol{x}}_{\imath}=\boldsymbol{u}\left(\boldsymbol{x}_{\imath}\right)+\frac{1}{6 \pi \mu a}\left(\boldsymbol{B}_{\imath}+T_{\imath} \hat{\boldsymbol{b}}_{\imath}-T_{\imath-1} \hat{\boldsymbol{b}}_{\imath-1}\right)
$$

involving the undetermined tensions. Applying the inextensibility constraint to the link between the $(i+1)$ th and $i$ th beads, $\left(\dot{\boldsymbol{x}}_{t+1}-\dot{\boldsymbol{x}}_{\boldsymbol{l}}\right) \cdot \boldsymbol{b}_{t}=0$, yields an equation with a tridiagonal matrix for the tensions

$$
\begin{aligned}
& T_{t+1} \hat{\boldsymbol{b}}_{\imath+1} \cdot \boldsymbol{b}_{\imath}-2 T_{\imath} \hat{\boldsymbol{b}}_{\imath} \cdot \boldsymbol{b}_{\imath}+T_{\imath-1} \hat{\boldsymbol{b}}_{t-1} \cdot \boldsymbol{b}_{\imath} \\
& \quad=\left(\boldsymbol{B}_{\imath+1}-\boldsymbol{B}_{\imath}\right) \cdot \boldsymbol{b}_{\imath}+6 \pi \mu a\left(u\left(\boldsymbol{x}_{\imath+1}\right)-u\left(\boldsymbol{x}_{\imath}\right)\right) \cdot \boldsymbol{b}_{\imath} .
\end{aligned}
$$

This equation can be applied for $i=1, \ldots, N-1$ with the convention that the end tension vanishes. Note that the above dynamics offers no resistance to untying a knot because it does not prevent one link passing through another.

It is convenient to non-dimensionalise the problem, scaling lengths with the length of the link, $b$, and scaling time with the time it takes an isolated bead to diffuse through the link length, $b^{2} / D=6 \pi \mu a b^{2} / k T$. Using this time-scale, the non-dimensional measure of the strain rate is the bead Péclet number or bead Weissenberg number $W_{\mathrm{b}}=\dot{E} b^{2} / D$. The total strain of the imposed flow after a time $t$, which in dimensional terms was $E=\dot{E} t$, now becomes in dimensionless variables $E=W_{\mathrm{b}} t$. The length and time scales give a scale for the velocity $k T / 6 \pi \mu a b$ which suggests a scale for the forces $k T / b$, with an association scale of $k T$ for the stresses. The scalings for the force and time yield the non-dimensional size of the random Brownian force $B=\sqrt{6 / \Delta t}$. Finally, the governing equation for the motion remains unchanged, except that the factor $6 \pi \mu a$ disappears, and the dimensional strain rate $\dot{E}$ is replaced by the non-dimensional flow strength $W_{\mathrm{b}}$. Henceforth all variables will be non-dimensional as described.

At each time-step, the imposed extensional flow at each bead and the random bead forces are evaluated. The tensions are then found which permit the velocity of each bead to be determined. A simple explicit first-order forward time-stepping was used, because the velocities change discontinuously through the random Brownian force.

Fig. 1 shows the evolution of an 800-link chain placed in the axisymmetric straining flow; in this case, Brownian motion has been neglected for economy, i.e. $W_{\mathrm{b}}=\infty$. Note that the initial random walk configuration is irregular and not the smooth hand sketch which carefully leaves no holes.

The size of the numerical time-step was initally taken to be $\Delta t=10^{-3}$ so that the typical random displacement of a bead was $\frac{1}{10}$. Such a large displacement meant that the length of the links typically increased by $1 \%$ at each time-step through second-order errors. The method chosen to correct this slow drift was to add to the above tensions a term proportional to the accumulated error in the length of the link, with a pseudo-spring strong enough to halve the error in three time-steps. 

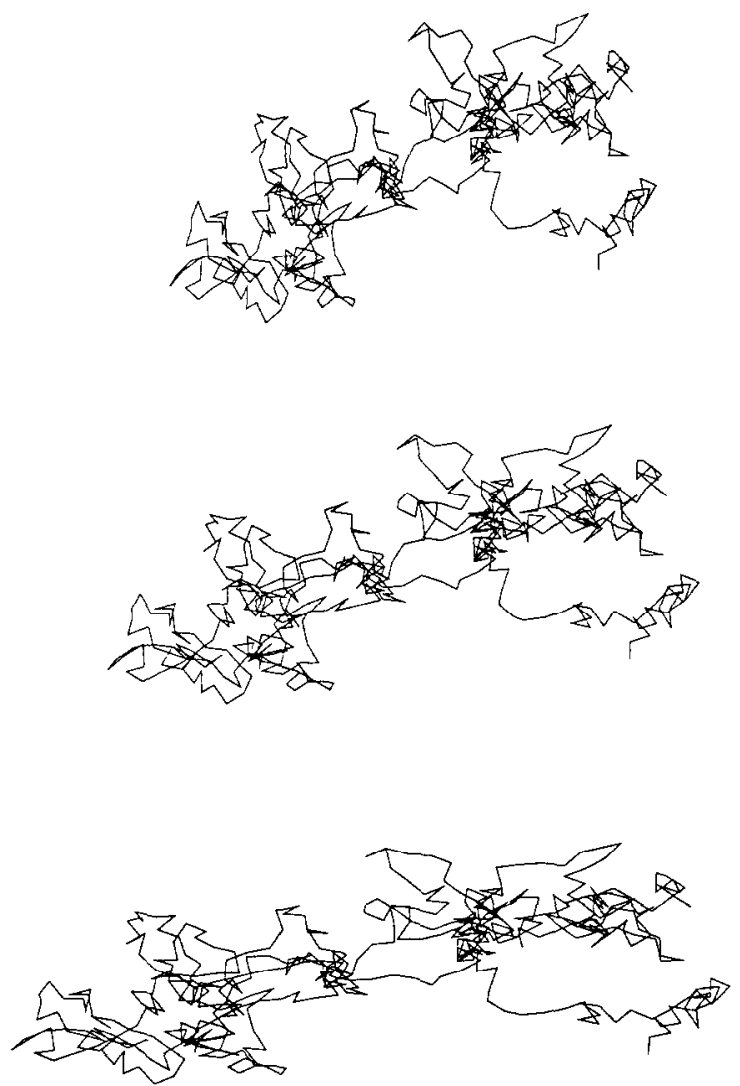

Fig. 1. A chain of 800 links placing the axisymmetric straining motion $\boldsymbol{u}(\boldsymbol{x})=\left(\dot{E} x,-\frac{1}{2} \dot{E} y,-\frac{1}{2} \dot{E} z\right)$ at total strains $E=W_{\mathrm{b}} t=0.0,0.2$ and 0.4 . There are no Brownan motions in this particular simulation.

Note that this problem cannot be avoided by just making the time-step smaller, because with random displacement $O\left(\Delta t^{1 / 2}\right)$ second-order errors will be $O(\Delta t)$ per time-step, and hence $O(1)$ after $O(1)$ time.

As the chain uncoiled, quite large tensions developed, proportional to the square of the extension. To avoid a numerical instability, it was found necessary to reduce the size of the time-step so that the largest tension acting alone could only displace a bead through a quarter of the length of a link. The maximum tension in the chain was therefore monitored, and the time-step halved when appropriate.

\subsection{Results for the radius of gyration}

The overall distortion is measured by the radius of gyration $R$ which is defined as

$$
R^{2}(t)=\frac{1}{N} \sum_{i=1}^{N}\left(x_{t}-\bar{x}\right)^{2} \quad \text { where } \bar{x}=\frac{1}{N} \sum_{i=1}^{N} x_{i}
$$




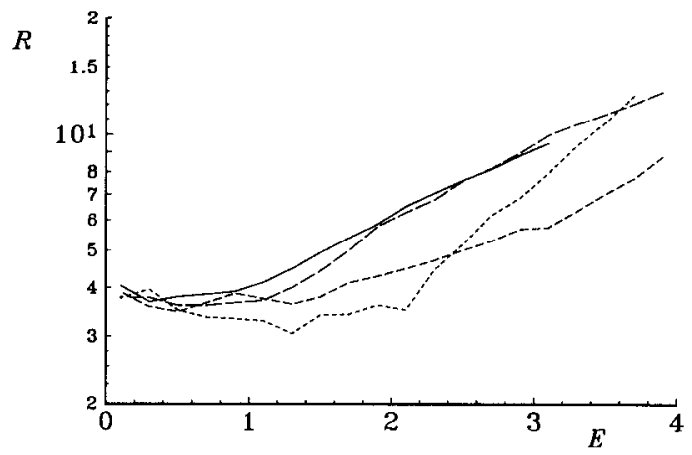

F1g. 2. The radius of gyration $R$ as a function of total strain $E=W_{\mathrm{b}} t$ for a chain of 100 links with the same initial configuration being placed in flows of different strengths: - - , $W_{\mathrm{b}}=0.01 ; \cdots, W_{\mathrm{b}}=0.02$; ,$--- W_{\mathrm{b}}=0.05 ;-, W_{\mathrm{b}}=0.1$.

This is a poor measure of small deformations, because it varies only quadratically with them, but at the large deformations of interest it is proportional to the overall linear size.

For a randomly coiled chain the (non-dimensional) radius of gyration is $R \approx$ $(N / 6)^{1 / 2}$ as $N \rightarrow \infty$. Thus for a typical simulation with $N=100, R$ is only 4.08 . If the coil becomes fully stretched, then $R \approx N /(12)^{1 / 2}$, which is 28.87 for $N=100$.

Fig. 2 shows the radius of gyration as a function of the total strain of the imposed flow for a chain of 100 links with the same initial configuration being placed in flows of different strengths. As all the flow have $W_{\mathrm{b}} \ll 1$, Brownian motion will dominate the advection by the flow for the individual beads. The collective diffusivity of all the beads together, however, is much weaker, being reduced by a factor of $1 / N$. Moreover, for the full chain to change its configuration, the collective diffusion must take place over the radius of gyration $O\left(b N^{1 / 2}\right)$. Thus one should define a Weissenberg number for the full chain as $W_{\mathrm{c}}=N^{2} W_{\mathrm{b}}$ (for these simula-

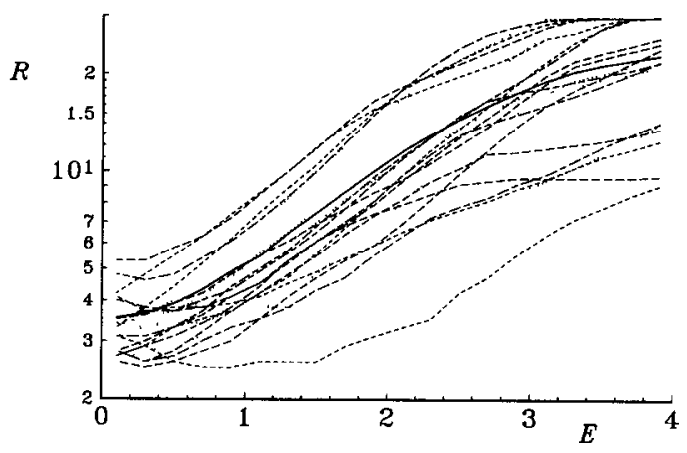

Fig. 3. The radius of gyration $R$ as a function of time $E=W_{\mathrm{b}} t$ for 22 different realisations of a chain with 100 links in a flow of strength $W_{\mathrm{b}}=0.2$. The continuous curve gives the arithmetic mean. 
tions which have no hydrodynamic interactions). A flow is then said to be strong (for the full chain) if $W_{\mathrm{c}}>1$. All the simulations in this paper are very strong in the sense that $W_{\mathrm{c}} \gg 1$.

There are large fluctuations between different realisations, as can be seen in Fig. 3 , which shows the growth of the radius of gyration as function of time for 22 realisations of a chain of 100 links. Note that at a total strain of $E=2.0$, one realisation is more than twice the size of the average, while another is less than half the size. Thus the accuracy of the average over 22 realisations is not good. Most of the results below will be for averages over fewer realisations.

Returning to the insensitivity of the gross distortions to Brownian motion when the flow strength is strong for the full chain $W_{\mathrm{c}} \gg 1$, Fig. 4 shows that the growth of the radius of gyration as a function of the total strain of the imposed flow, when averaged over several realisations, is independent of the magnitude of the strain rate as long as it is strong. Fig. 5 shows the configurations at three different times for two chains which started from the same initial configuration, one in a strong flow and the other with no Brownian motion. It can be seen quite clearly that the gross distortion is the same. However, the Brownian motions are dominant at the scale of the single link, producing large fluctuations of the chain at the small scale.

In strong flows, the dumbbell model would have the advected solution

$$
R^{2}(t)=\frac{N}{6} \frac{\mathrm{e}^{2 W_{\mathrm{b}} t}+2 \mathrm{e}^{-W_{\mathrm{b}} t}}{3}
$$

until finite length effects become important This is also plotted in Fig. 4. It is seen that the short chains with $N=100$ extend more slowly, being decelerated by the unfolding of the back-loops. Longer chains are advected by the flow more efficiently, until they become almost fully extended.

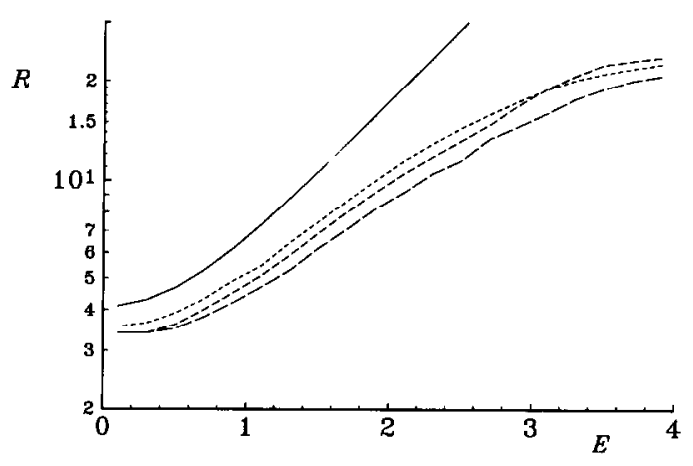

Fig. 4. The radius of gyration $R$ for a chain of 100 links averaged over several realisations as a function of the total strain $E$ at various strong flow strengths:,$--- W_{\mathrm{b}}=0.2$ (22 realisations);--,$W_{\mathrm{b}}=1.0(4$ realisations); - - - no Brownian motions ( 9 realisations). Also plotted with the solid curve is the advection solution for strong flows. 

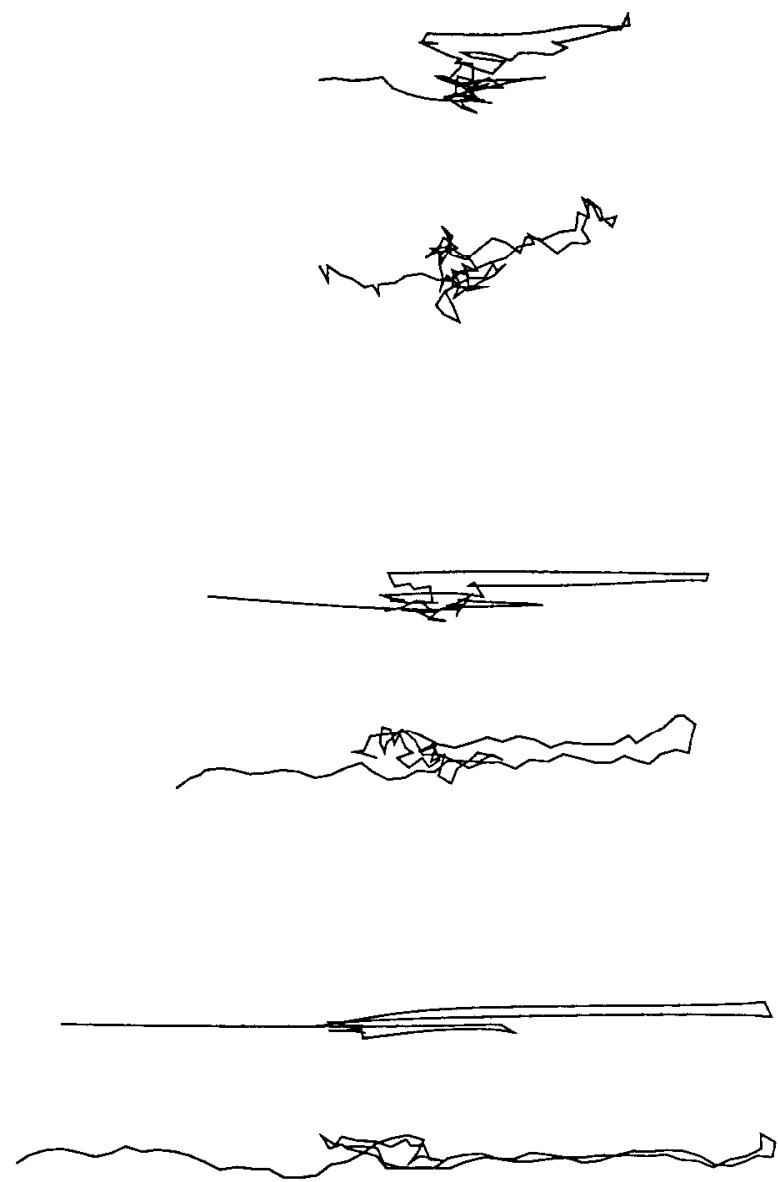

Fig. 5. The configurations at total strains $E=W_{b} t=0.8,1.6$ and 2.4 for a chain with 100 links, with no Brownian motion and with $W_{\mathrm{b}}=0.2$, both chains starting from the same initial conditions.

\subsection{Results for the stress}

The contribution of one chain to the stress tensor $\sigma$ is the moment of the Brownian and tension forces, which in light of the force balance is equal to the moment of the hydrodynamic forces:

$$
\sigma=\sum_{t=1}^{N} F_{l} x_{l}
$$

The non-dimensionalisation takes a scale of $k T$ for the stress. In the axisymmetric straining motion, one is interested in the deviatoric stress:

$$
\sigma^{\prime}=2 \sigma_{11}-\sigma_{22}-\sigma_{33}
$$


In thermodynamic equilibrium with no flow, the stress tensor has an averge value of $N I$. There are, however, large fluctuations at each instant for a single realisation, essentially fluctuations of the order $B R \sqrt{N}$. Thus the fluctuations dominate the average in the unstressed state by a factor of $O(1 / \sqrt{\Delta t})$. Such large fluctuations were reduced by one order of magnitude by averaging the stress over the small interval of time during which the total strain changes by 0.2 , an interval typically containing some 1000 time-steps. A long interval could not be used because the system is evolving. Further averages were made over several realisations. However, the fluctuations remain comparable with the average value of the unstressed state. ${ }^{1}$

The large fluctuations in the stress, even averaged over 1000 time-steps, are seen in Fig. 6. Each realisation shows larger fluctuations with time for the smaller strains, sometimes resulting in negative values. At larger strains, the fluctuations become relatively smaller for each realisation, although there are very large differences between different realisations.

Also shown in Fig. 6 is the average over 11 realisations. Its magnitude is $O(N)$ and grows with $R^{2}$ roughly according to the elastic dumbbell theory, which has $\sigma^{\prime} \sim 36 R^{2}$ in sudden large deformations.

When the flow rate was increased by a factor of five, however, the stress was also found to increase by the same factor. This is not the response of an elastic dumbbell, which would have the same stress for the same total stain - recall that the gross distortion as measured by the radius of gyration was found to depend on the total strain, but to be independent of the value of the strain rate once that was large. Fig. 7 shows that if $W_{\mathrm{c}} \gg 1$ the stress can instead be characterized by an extensional viscosity (non-dimensionalised by $6 \pi \mu a b^{2}$ )

$$
\mu_{\mathrm{ext}}=\sigma^{\prime} / 6 W_{\mathrm{b}}
$$

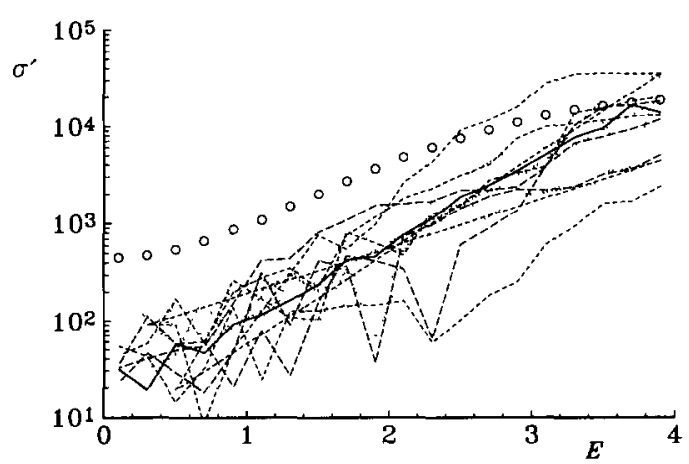

Fig. 6. The deviatoric stress $\sigma^{\prime}$ as a function of time $E=W_{b} t$ for 11 different realisations of a chain with 100 links, with $W_{\mathrm{b}}=0.2$. The average is given by the solid curve, while the open circles give the elastic dumbbell stress $36 R^{2}$

\footnotetext{
${ }^{1}$ Grassia [13] has recently developed a superior algorithm for the stress which avoids the $O(1 / \sqrt{\Delta t})$ fluctuations.
} 


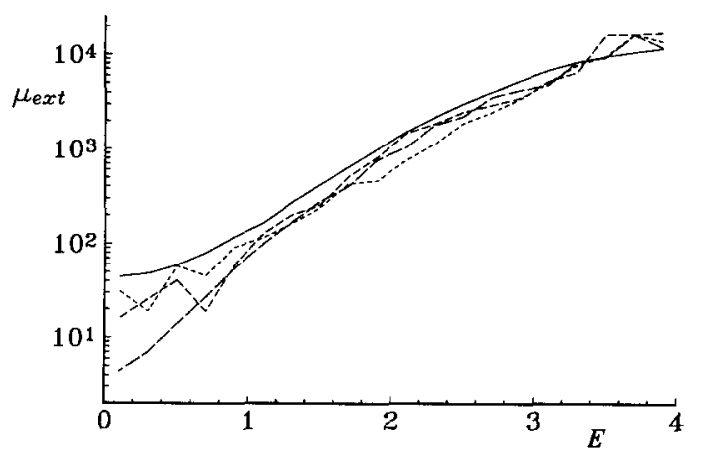

Fig. 7. The extensional viscosity $\mu_{\text {ext }}$ for a chain of 100 links, averaged over several realisations, as a function of the total strain $E$ at various flow strengths: - - , $W_{\mathrm{b}}=0.2\left(11\right.$ realisations);----, $W_{\mathrm{b}}=1.0$ (four realisations); - - - with no Brownian motions (nine realisations). The solid curve is $R^{3}(t)$.

which is independent of the strain rate, although a rapidly increasing function of the total strain. For fully stretched chains, this extensional viscosity takes the limiting value $\frac{1}{36} N^{3}$.

There is some experimental support for the stress being dissipative rather than elastic in strong straining motions, as found in the simulations. ${ }^{2}$ Moan and Magueur [14] found in an open syphon experiment that the stress depended strongly on the deformation up to the syphon, and that the results at different flow rates could be superposed by plotting the local stress divided by the local strain rate, i.e. a local viscosity, as a function of the total strain. Similarly, in a filament stretching device, Tirtaatmadja and Sridhar [15] found a universal behaviour by plotting the stress divided by the strain rate as a function of total strain, a universal behaviour for each material and for all sufficiently high strain rates.

The frozen rigid model of King and James [9] and the yo-yo model of Ryskin [10] both suggest that the stress would be characterised by an extensional viscosity, and that this viscosity should increase with the cube of the radius of gyration. The computer simulations for $N=100$ do have an extensional viscosity which increases roughly with $R^{3}(t)$ as shown by the solid line in Fig. 7. However, the coefficient of proportionality displayed in Fig. 8 is not found to be an $O(1)$ constant as the number of links in the chain $N$ is increased, but instead the coefficient of proportionality decreases with increasing $N$. Fig. 9 shows that as the length of the chain increases it seems that the contribution of each link to the viscosity does not change. In a certain sense this means that the overall size of the chain is irrelevant and each link creates a stress interacting with its local neighbours. This unexpected, and here tentative, conclusion will be explained by the 'kinks dynamics' model later.

\footnotetext{
${ }^{2}$ It is possible that the conclusion for the simulations that the elastic stresses are small is wrong, and is a result of the crude algorithm used to calculate the stress.
} 


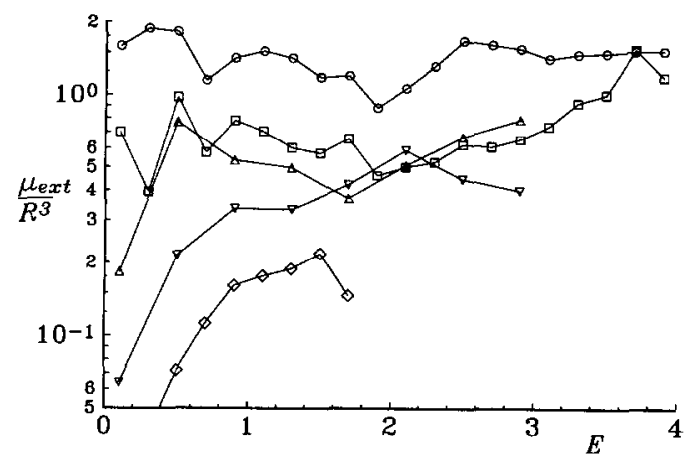

Fig. 8. The cxtensional viscosity $\mu_{\text {ext }}$ divided by $R^{3}$ (the scaling of the yo-yo model) as a function of the total strain $E$ for different numbers of links $N: O, N=50\left(W_{\mathrm{b}}=0.2,11\right.$ realisations), $\square, N=100$ ( $W_{\mathrm{b}}=0.2,11$ realisations); $\triangle, N=200$ ( $W_{\mathrm{b}}=0.4$, three realisations); $\nabla, N=400$ ( $W_{\mathrm{b}}=0.4$, five realisations $) ; \diamond, N=800\left(W_{\mathrm{h}}=0.4\right.$, four realisations).

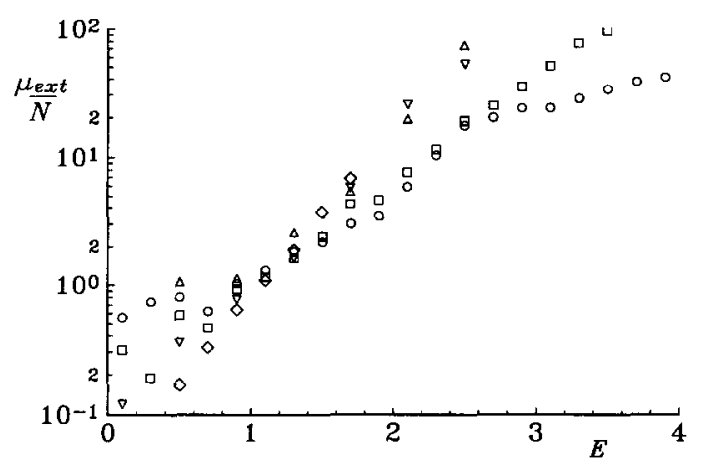

Fig. 9. The viscosity per link $\mu_{\mathrm{ext}} / N$ as a function of the total strain $E$ for chains with different numbers of links; symbols as for Fig. 8.

\subsection{Results for birefringence}

Birefringence measures the average orientation of the links, $\hat{b}_{i}$ which have unit length. For the axisymmetric straining motion, one is interested in the deviatoric part

$$
b^{\prime}=\frac{1}{N} \sum_{i=1}^{N-1} 2 \hat{b}_{l 1}^{2}-\hat{b}_{i 2}^{2}-\hat{b}_{l 3}^{2} \text {. }
$$

This varies from 0 for random orientations to 2 when all the links are fully aligned with the flow. Note that it is possible for all the links to be aligned with the flow without the chain being fully stretched, as will occur with back-loop configurations. Thus a stress-optical law may have a limited applicability, particularly in this transient strong flow.

Fig. 10 shows the evolution of the birefringence with the total strain of the imposed flow, averaging the value over the short interval of 0.2 of total strain and 


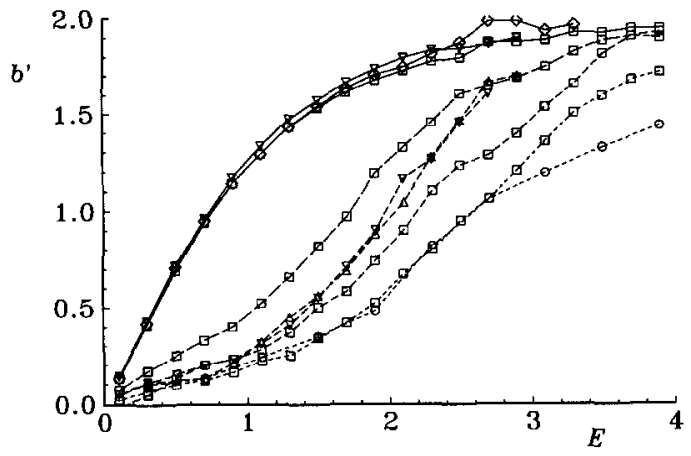

Fig. 10. The birefringence $b^{\prime}$ as a function of the total strain $E$ with different lengths of chain and different flow strengths: $O, N=50 ; \square, N=100 ; \Delta, N=200 ; \nabla, N=400 ; \diamond, N=800 ;--, W_{\mathrm{b}}=0.2$; ,$--- W_{\mathrm{b}}=0.4 ;-\ldots-, W_{\mathrm{b}}=1.0 ;-$, no Brownian motions.

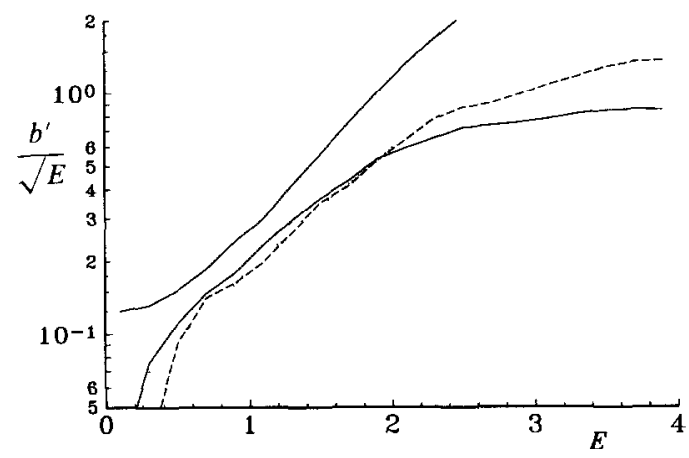

Fig. 11. The birefringence $b^{\prime}$ divided by $\left(W_{\mathrm{b}}\right)^{1 / 2}$ for a chain of 100 links as a function of total strain for different flow strengths: $\cdots, W_{\mathrm{b}}=0.2 ; \cdots, W_{\mathrm{b}}=0.4 ;-\cdots, W_{\mathrm{b}}=1.0$. The solid line is proportional to $R^{2}(t)$.

over several realisations. It can be seen that the birefringence depends on the strain rate and on the total strain, but that the average alignment per link is independent of the length of the chain. Again, the dynamics exhibits a local behaviour rather than one depending on co-operative effects over the full length of the chain.

Fig. 11 shows that at intermediate strains the birefringence appears to increase roughly as $R^{2}(t)$, and over the limit range of $0.2 \leq W_{\mathrm{b}} \leq 1.0$ is proportional to $\left(W_{\mathrm{b}}\right)^{1 / 2}$. These correlations are not understood.

\section{Kinks dynamics}

\subsection{Governing equations}

As the polymer chain extends it becomes more of a one-dimensional structure The key to the scaling as $N \rightarrow \infty$ for the increase of stress with time is an 
understanding of the growth of the straightened segments. To examine their dynamics, to see how they grow in length and reduce in number as they unfold, we now look at a highly simplified one-dimensional model, the kinks dynamics model, which has already been studied by Larson [11]. This model has no Brownian motion.

We take as the initial configuration a random walk in one dimension with $N$ steps of fixed length $b$. This initial state of folded straight segments is then evolved as if it were a fully flexible string, an inextensible string, in the flow $u=\dot{E} x$. Typically, there will be $N / 2$ straight segments in the initial configuration. As the string unfolds with time, the number of segments will decrease.

Let the $i$ th straight segment run from $x_{2}(t)$ to $x_{i+1}(t)$. Now, because the string is inextensible (exerting a tension to stop any stretching), the segment will move at the velocity of the flow averaged along its length:

$$
v_{l}(t)=\frac{1}{2} \dot{E}\left(x_{l+1}+x_{l}\right)
$$

We ignore here any hydrodynamic interactions between the different segments. We also assume that adjacent segments exert no force through the junctions, which is appropriate for a flexible string that cannot support compression. (An attempt to speed up the three-dimensional simulations by treating some straight segments as being rigidly locked was unsuccessful at producing a speed up, but did find that there was very little tension near the ends of the straight segments.)

Because adjacent segments move at different velocities, the lengths of the segments will grow and contract. Let the junction of the $(i-1)$ th and $i$ th segments be at the arc length distance $s_{l}(t)$ from the beginning of the string. For a segment with a forward direction, $x_{i+1}>x_{i}$, the position of the $i$ th junction will move at velocity $\dot{s}_{t}$ relative to the material points on the $i$ th segment, and at $-\dot{s}_{\imath}$ relative to the material points on the previous $(i-1)$ th segment (which has the reverse direction $\left.x_{\imath}<x_{\imath-1}\right)$, i.e.

$$
v_{\imath}+\dot{s}_{i}=v_{\imath} \quad 1-\dot{s}_{\imath} \text {. }
$$

Using the geometry $x_{t+1}=x_{t}+\left(s_{t+1}-s_{i}\right)$ and $x_{t}=x_{t-1}-\left(s_{i}-s_{t-1}\right)$ for this forward-reverse pair, we obtain the evolution equation

$$
\dot{s}_{t}=\frac{1}{4} \dot{E}\left(-s_{t+1}+2 s_{t}-s_{t-1}\right) \text {. }
$$

The identical equation is obtained for the opposite reverse-forward pair of segments. It is easy to integrate these equations forward in time starting from the initial conditions on $s_{t}(0)$.

As the system evolves, the small segments are consumed by adjacent larger segments. This occurs when $s_{t}(t)$ becomes equal to $s_{t+1}(t)$ for some $i$. At that moment the $i$ th segment disappears. It is then necessary to relabel all the segments further along the string.

While the original system of equations can be integrated analytically, it is the abrupt relabelling which makes the problem better suited to numerical simulations. As these simulations are very much simpler than the full three-diemsional simulations, it was possible to make 100 realisations with different initial configurations 


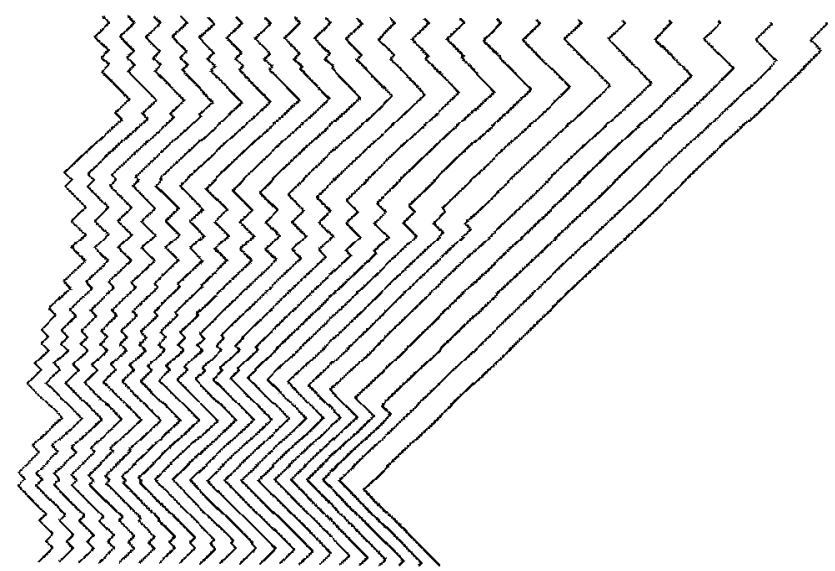

Fig. 12. A simulation of the kink dynamics with $N=80$. The interval in time between the configurations is $0.1 / \dot{E}$.

using $N=20,40,80,160,320,640,1280$ and 2560 . This range allows scaling with $N$ to be explored.

A typical simulation with $N=80$ is shown in Fig. 12. The one-dimensional walk in the horizontal direction has been opened up vertically so that it can be seen, the vertical position having no dynamic significance. The time between each displayed configuration is $0.1 / \dot{E}$, so that the total strain at the last configuration is $\dot{E} t=2.0$. The larger segments grow at the expense of the smaller ones.

\subsection{Results for the radius of gyration and the stress}

The size of the unfolding chain can be measured by its radius of gyration defined as for the three-dimensional chain. The initial random configuration has a radius of

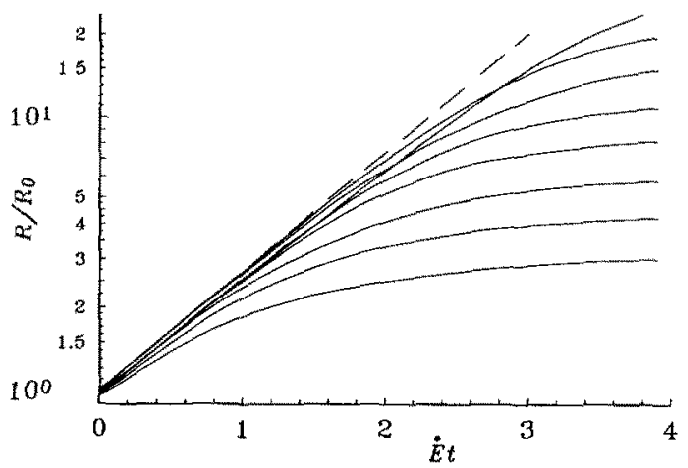

Fig. 13. The radius of gyration $R(t)$ divided by the initial value $R_{0}=(N / 6)^{1 / 2}$ as a function of the total strain $\dot{E} t$, averaging over 100 realisations for each chain length $N=20,40,80,160320,640,1280,2560$. The dashed curve corresponds to advection with the flow $R(t)=R_{0} \mathrm{e}^{E t}$. 
gyration of $R_{0}=b(N / 6)^{1 / 2}$. The growth in the size of the chain given by $R(t) / R_{0}$ is plotted in Fig. 13 as a function of the total strain $\dot{E} t$. Each curve corresponds to the average over 100 realisations for the different values of $N$ between 20 and 2560 . It can be seen that the chains are sufficiently deformable to be advected with the flow, i.e. $R=R_{0} \mathrm{e}^{\dot{E} t}$ as given by the dashed curve, until they are within a third of being fully stretched (where $R=b N /(12)^{1 / 2}$ ). In the final stages the chain becomes more rigid, with a segment stretching the full width of the chain. The unfolding of the last few back-loops is relatively slow, although only finite time is taken by any individual chain.

The contribution of a chain to the stress tensor in the kinks dynamics model is dissipative, corresponding to the viscous dissipation as each segment of inextensible string fails to deform with the flow. This can be represented by an extensional viscosity contribution:

$$
\mu_{\mathrm{ext}}=\mu \sum \frac{1}{12}\left(s_{i}-s_{2}\right)^{3} .
$$

Fig. 14 gives the growth of the extensional viscosity contribution with time. It was found that the behaviour of the chains of different lengths could be brought onto a single curve (while they were less than a third fully stretched) by plotting the extensional viscosity divided by the numer of initial links (and a dimensional factor of $\mu b^{3}$ ). For the initial random walk, one can calculate the expectation of the size of the segments to be $2 b$, along with the expectation of the square and cube of the size as $6 b^{2}$ and $26 b^{3}$. Thus one can predict that the initial extensional viscosity contribution is $13 \mu b^{3} / 12$, which was found in the simulations. In the fully stretched state the contribution becomes $\mu N^{3} b^{3} / 12$. Fig. 14 shows that the stress grows rapidly with time, more rapidly than the $R^{3}(t)$ behaviour of the yo-yo model. This rapid growth comes from events in which two segments merge into one that is roughly twice as large, giving four times the viscous dissipation. We interpret this rapid growth in the dissipation as the deformable chain becomes more rigid in nature as it unfolds.

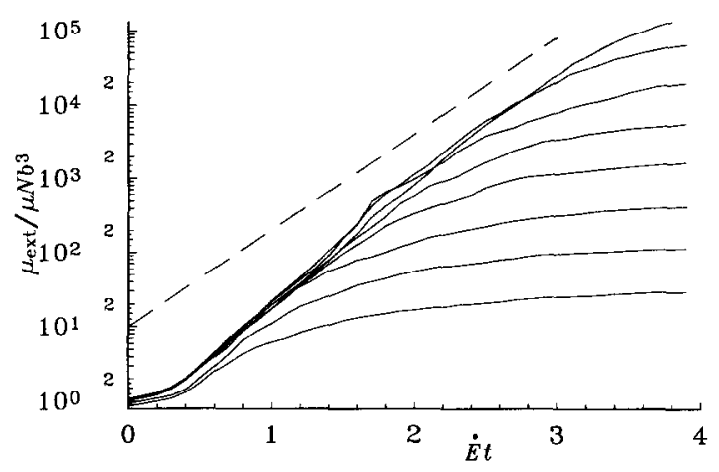

Fig. 14. The extensional viscosity contribution per link, $\mu_{\mathrm{exi}} / \mu b^{3} N$, as a function of the total strain $\dot{E} t$, averaged over 100 realisations for each of the chain lengths $N=20,40,80,160,320,640,1280$ and 2560 . 


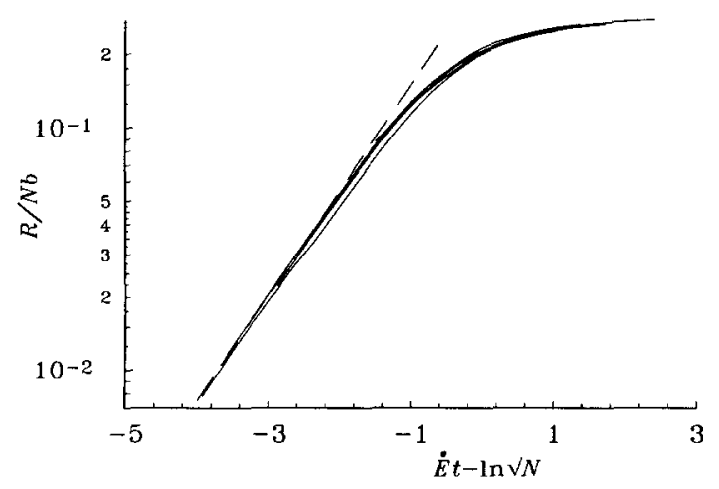

Fig. 15. The radius of gyration $R$ divided by the full length of the chain $N b$ as a function of the total strain relative to that required to fully stretch the chan, $\dot{E} t-\ln \sqrt{N}$.

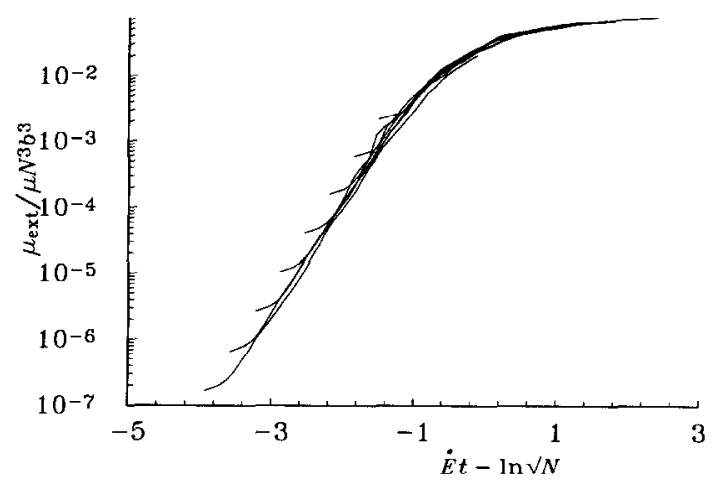

Fig. 16. The extensional viscosity contribution $\mu_{\text {ext }}$ divided by $\mu N^{3} b^{3}$ as a function of the total strain relative to that required to fully stretch the chain, $\dot{E} t-\ln \sqrt{N}$.

Figs. 13 and 14 give the growth in size and the dissipation from the perspective of the initial chain. Larson [11] showed that the final stages, as the chain becomes fully stretched, have a universal behaviour independent of the length of the chain. To see this universal behaviour, time is mesured as the total strain relative to the value required to advect the initial random walk to the fully stretched state, i.e. as $\dot{E} t-\ln \sqrt{N}$. Similarly, the radius of gyration and dissipation are scaled by $N$ and $N^{3}$ respecively, as appropriate for the fully stretched chain. Figs. 15 and 16 give further evidence of Larson's universal behaviour in the final stages of unfolding.

\subsection{Simple scaling of the unfolding}

To obtain some insight into the unfolding process, we can examine how the number of straight segments decreases with time as their typical length increases. In Fig. 17 we plot the number of segments, $n_{\mathrm{s}}$, divided by the number of initial links $N$ as a function of the total strain $\dot{E} t$. The number of segments for the initial 


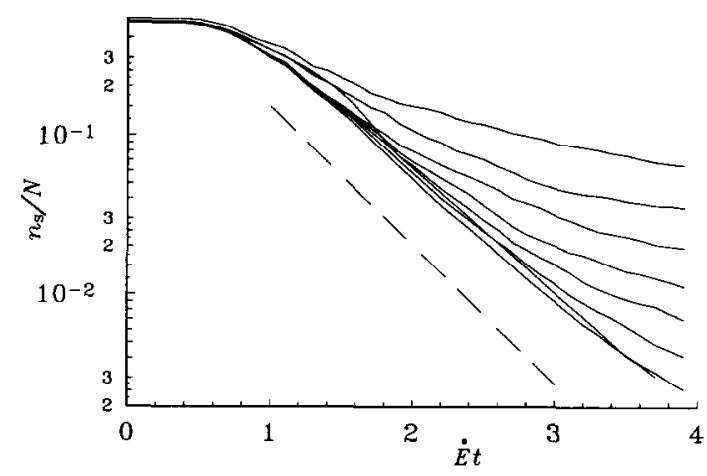

Fig. 17. The number of segments $n_{\mathrm{s}}$ divided by the number of initial links $N$ as a function of the total strain $\dot{E} t$. The dashed curve corresponds to $\mathrm{e}^{-2 E t}$

random walk is $\frac{1}{2} N$. After the first unit of strain, the number of segments seems to decrease exponentially approximately as $e^{-2 E t}$, until the chain is nearly fully stretched.

To study the evolution of the lengths of the segments we consider the distribution of the lengths at different times. There is a statistical problem of maintaining a sufficiently large sample when dividing the segments into groups of different length, particularly for the rarer longer segments. An adequate compromise was found to count the number of segments $v(\Delta s, t)$ with lengths $\Delta s$ in the range $\left[2^{k / 2}, 2^{(k+1) / 2}\right]$. Fig. 18 gives this distribution at the total strains $\dot{E} t=1.0$ and 2.0. It was found posisble to bring together the results for the chains of different lengths by dividing the number of segments $v$ by the number of initial links $N$. This scaling does not work as the chains become fully stretched as seen at $t=2.0 / \dot{E}$, when the results for the short chains $N=20$ and 40 separate from the remainder. At the later time of $3.0 / \dot{E}$ (not shown), the chains with $N=80$ also deviate from all the longer chains.

A replot of the distributions on log-linear scales reveals that the longest segments have an exponential distribution, $v(\Delta s, t) \propto 2^{-\Delta s}$ as $\Delta s \rightarrow \infty$, like that for the initial random walk. After the first unit of strain which is dominated by the distribution of the initial random walk, a rather flat distribution emerges, spanning $2 \leq \Delta s \leq 40$ at $t=2.0 / \dot{E}$ and $10 \leq \Delta s \leq 300$ at $t=3.0 / \dot{E}$. Thus, while it is difficult to give a precise definition of the typical length, it is clear that it is growing rapidly.

The evidence in Figs. 17 and 18 indicates that, until the chain is nearly fully stretched, each part of the chain evolves in a similar way. One could cut the chain into several pieces without causing a significant decrease in the number of segments with time and their distribution. The chain is therefore sufficiently deformable and non-rigid that the dynamics are controlled locally: until the chain is nearly fully stretched, the unfolding process does not 'feel' the total length of the chain.

It is appropriate here to recall the results in Fig. 9 and 10 for the three-dimensional simulations in Section 2. These figures show that the contribution to the viscosity and to the birefringence from each link was independent of the number of links in the chain. 

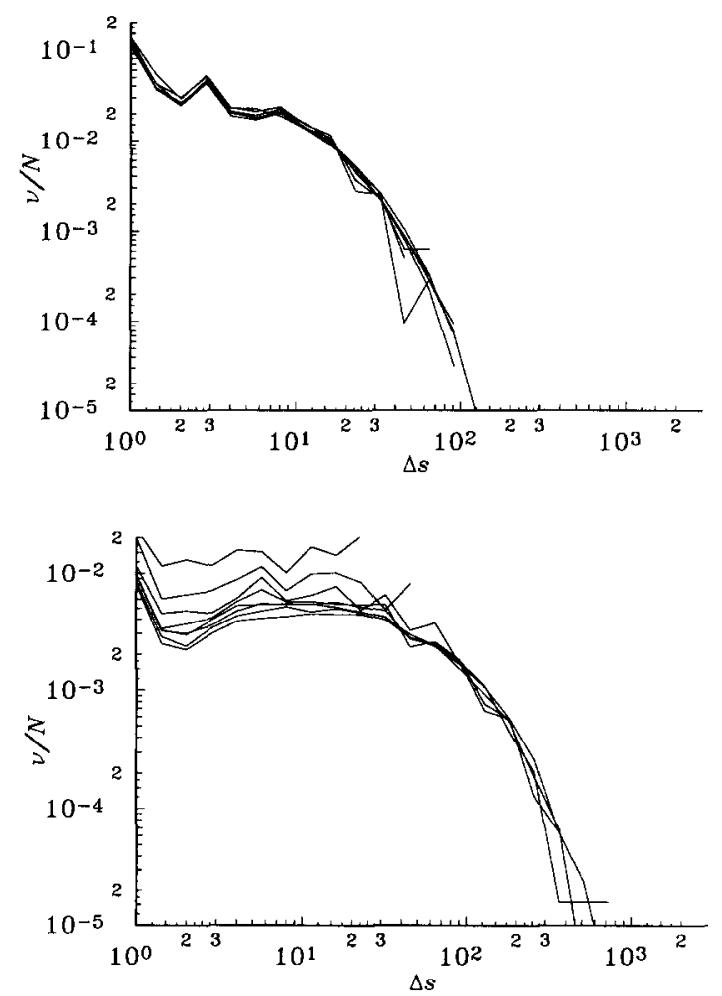

Fig. 18. The distribution of the length of the segments at the total strains $\dot{E} t=1.0$ and 2.0. The number of segments of lengths $\Delta s$ in the range $\left[2^{k / 2}, 2^{(k+1) / 2}\right]$ is denoted by $v(\Delta s, t)$.

It has not proved possible to start from the growing differential equations and then derive an estimate of the growth of the typical length of the segments. The governing equation gives a large segment which grows fastest when it is surrounded by two smaller ones, then growing as $\mathrm{e}^{\dot{E} t / 2}$, which is far too slow. The rapid growth occurs instead when a small segment is fully consumed and two larger segments combine suddenly to form one twice as large. Quantifying this growth process is a more difficult task. All that can be easily deduced from the governing equtions is that the rate of growth is proportional to the current length and so the typical length should grow exponentially with time.

Fortunately, progress can be made from the ideas that until it is nearly fully stretched the chain is very deformable and that one part of the chain is quite independent of a remote part. If $l_{\mathrm{s}}(t)$ is the typical length of the $n_{\mathrm{s}}(t)$ segments, the conservation of the total chain length gives

$$
n_{\mathrm{s}}(t) l_{\mathrm{s}}(t)=N b \text {. }
$$

A second relationship comes from estimating the radius of gyration $R$ as the size of a random walk of $n_{\mathrm{s}}$ independent steps of size $l_{\mathrm{s}}$ :

$$
R(t)=k\left(n_{\mathrm{s}}(t)\right)^{1 / 2} l_{\mathrm{s}}(\mathrm{t})
$$


The $O(1)$ constant $k$ depends on the details of the distribution of the segment lengths and will change with time. For the initial random walk, $k=\frac{1}{12}$. Finally, the high deformability of the chain means that the radius of gyration will grow by being advected with the flow

$$
R(t)=R_{0} \mathrm{e}^{\dot{E} t}
$$

Solving, we obtain the estimates

$$
n_{\mathrm{s}}(t)=6 k N \mathrm{e}^{-\dot{E} t} \quad \text { and } l_{\mathrm{s}}(t)=\frac{1}{6 k} b \mathrm{e}^{2 \dot{E} t} .
$$

Fig. 17 shows this rate of exponential decrease in the number of segments while the chains are not nearly fully stretched. After the first unit of strain, it appears that the value of the constant $k=\frac{1}{2}$ would be appropriate.

To test the predictions of the growth of the lengths of the segments we have plotted in Fig. 19 the distribution of the lengths of the segments, multiplying the number of segments by $\mathrm{e}^{2 E t} / N$ and their length by $\mathrm{e}^{-2 E t}$. It is seen that with these scalings the different times and different chain lengths are superposed.

From the above estimates of the number and length of the segments, we can proceed to an estimate of the extensional viscosity (while the chain is unfolding)

$$
\mu_{\text {ext }}=\mu \frac{k_{2}}{12} n_{\mathrm{s}} l_{\mathrm{s}}^{3}=\mu N b^{3} \mathrm{e}^{4 \dot{E} t} \frac{k_{2}}{432 k^{2}},
$$

with a further $O(1)$ constant $k_{2}$ which depends on the distribution of segment lengths, and will therefore change with time. For the initial random walk, $k_{2}=13 / 4$. Note that the extensional viscosity grows proportionally to $\mathrm{e}^{4 \dot{E} t}$, more rapidly than the $\mathrm{e}^{3 E t}$ of the $R^{3}(t)$ in the yo-yo model, as seen earlier in Fig. 14. In Fig. 20 we have plotted $\mu_{\text {ext }} N b^{1} / \mu R^{4}(t)$, which the above estimates give as $k_{2} / 12 k^{2}$. We see that the results for the different chains are superposed with the constant $k_{2}$, increasing from its initial value of $13 / 4$ to about 45 , i.e. $k_{2} / 12 k^{2}$ decreasing from 39 to 15 , during the

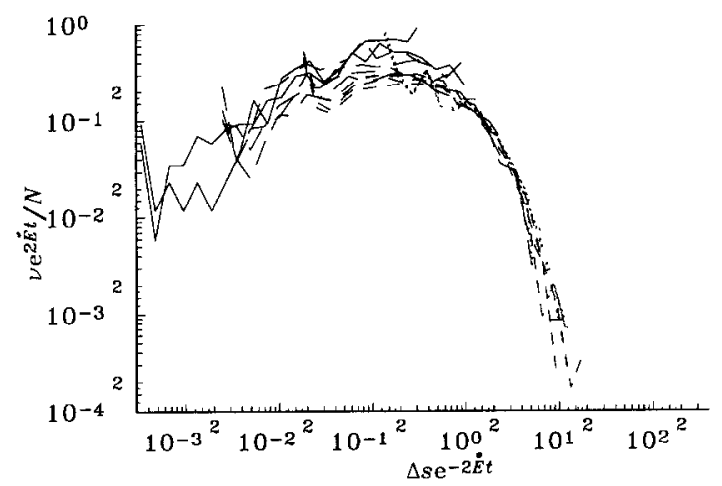

Fig. 19. The scaled distribution of the scaled lengths of the segments, scaling the number with $N \mathrm{e}^{-2 \dot{E} t}$ and their length with $\mathrm{e}^{2 E t}: \quad, \dot{E} t=4.0 ; \cdots, \dot{E} t=3.0 ; \cdot, \dot{E} t=2.0$. The results are for the longest chains $N=640,1280$ and 2560 . 


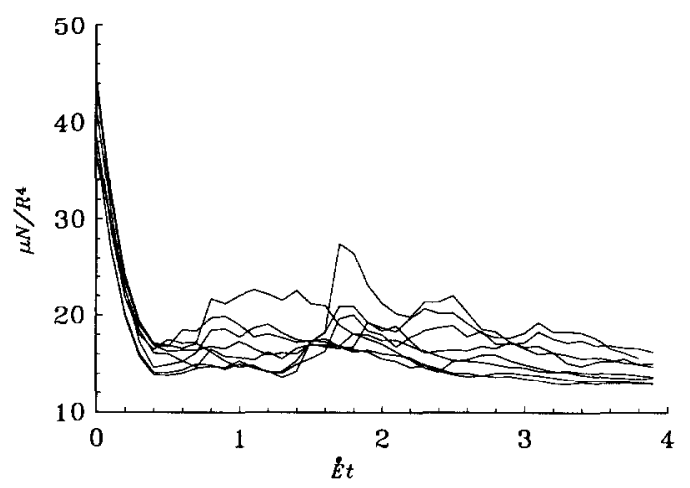

Fig. 20. The extensional viscosity $\mu_{\text {ext }}$ divided by $R^{4}(t) / N b^{4}$ as a function of the total strain $\dot{E} t$.

first unit of strain and then remaining constant. We would not have found such a universal behaviour with the yo-yo model scaling $\mu_{\text {ext }} b^{3} / R^{3}(t)$. While the above estimates are for an unfolding chain which is not nearly fully stretched, using the radius of gyration in place of the exponential $e^{E t}$ yields an expression which can be applied to the fully stretched chain. We note that $\mu N b^{4} / R^{4}$ for a fully stretched chain is 12, and this is seen to be approached in Fig. 20. One possible interpretation of the $R^{4}$ scaling of the extensional viscosity is to consider the dissipation as arising from a particle of size $R$ with a rigidity which increases as $R(t) / N b$.

\section{Conclusions}

The three-dimensional simulations of Section 2 studied an isolated polymer chain uncoiling in a suddenly applied very strong extensional flow. The stress was found to increase rapidly with time and is shown in Fig. 7 to be dissipative in nature rather than elastic, ${ }^{3}$ i.e. proportional to the instantaneous strain rate rather than being independent of it. The increase was shown in Fig. 8 to follow roughly the yo-yo scaling of being proportional to $R^{3}(t)$, but in disagreement with the yo-yo model the constant of proportionality decreased with increasing $N$ as the longer chains behaved less rigidly.

The kinks dynamics of Section 3 showed how the rigidity of the chain varies as $R(t) / N b$, i.e. starts very flexibly like $N^{-1 / 2}$ and increased to fully rigid. The yo-yo model with two coiled ends joined by a fully stretched central region was not seen in the three-dimensional simulations. Instead there was a gradual buildup of many fully stretched segments. The kinks dynamics model showed that the number of straight segments decreased as $N^{2} b^{2} / R^{2}(t)$ (see Fig. 17) as their typical length increased as $R^{2}(t) / N b$, (see Fig. 19). This leads to the prediction that the dissipative stress build up with time as $R^{4}(t) / N$ (see Fig. 20).

\footnotetext{
${ }^{3}$ It is possible that this conclusion that the elastic stresses are small is wrong, and is a result of a crude algorithm used to calculate the stress.
} 


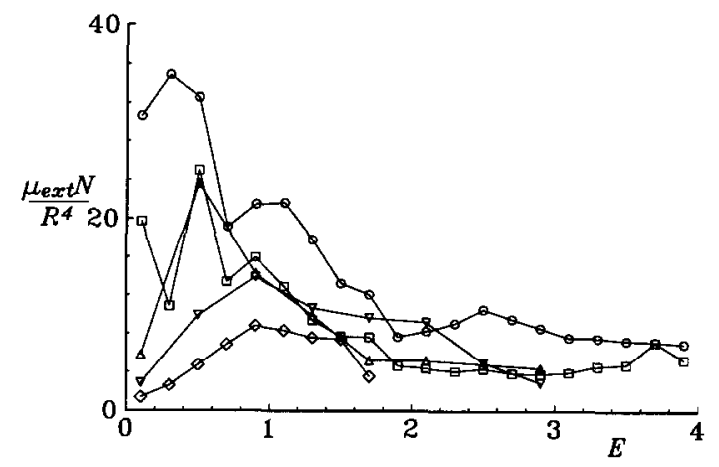

Fig. 21. The extensional viscosity $\mu_{\text {ext }}(t)$ divided by $R^{4}(t) / N$ (the kinks scaling) as a function of the total strain $E$ for the three-dimensional simulations with different numbers of links $N: O, N=50\left(W_{\mathrm{b}}=0.2\right.$, 11 realisations); $N: \square, N=100$ ( $W_{\mathrm{b}}=0.2,11$ realisations); $N: \Delta, N=200\left(W_{\mathrm{b}}=0.4\right.$, three realisations); $N: \nabla, N=400$ ( $W_{\mathrm{b}}=0.4$, five realisations); $N: \vartheta, N=800$ ( $W_{\mathrm{b}}=0.4$, four realisations).

We must now test whether the kinks scaling for the stress is applicable to the three-dimensional simulation of Section 2. In Fig. 21 the data of Fig. 8 have been replotted, now plotting $\mu_{\text {ext }}(t) N / R^{4}(t)$ as a function of the total strain $E$. We see that, unlike in Fig. 8, there is no longer a systematic decrease in the constant of proportionality as $N$ increases. Moreover, the value of $\mu_{\mathrm{ext}} N / R^{4}$ in Fig. 21 hovers around 15, the eventual value in Fig. 20 for the one-dimensional kinks model. The only difference between the three- and one-dimensional simulations seems to be at the early times, when the total strain is less than unity, where the long three-dimensional chains seem to be a little more flexible.

We now need to encapsulate the above stress behaviour in a simple constitutive equation. The simplest model of the gross distortion of a polymer is the elastic dumbbell which leads to the Oldroyd-B fluid with $f \equiv 1$

$$
\begin{aligned}
& \sigma=p \boldsymbol{I}+2 \mu \boldsymbol{E}+G f \boldsymbol{A} \\
& \frac{\mathrm{D} \boldsymbol{A}}{\mathrm{D} t}=\boldsymbol{A} \cdot \nabla U+\nabla U^{T} \cdot \boldsymbol{A}-\frac{f}{\tau}(\boldsymbol{A}-\boldsymbol{\eta}),
\end{aligned}
$$

with solvent viscosity $\mu$, elastic modulus $G$ and relaxation time $\tau$. Here the deforming microstructure is represented by the second-order tensor $\boldsymbol{A}$, which can be thought of as the moment of inertia tensor of the chain divided by its equilibrium value $\mathrm{Nb}^{2} / 6$. The Oldroyd-B fluid suffers from infinite and negative values of the steady extensional viscosity when the strain rate exceeds $1 / 2 \tau$. This problem arises because its microstructure can deform without bound. The problem can be cured by restricting the deformation of the elastic dumbbell with a FENE modification in which

$$
f=\frac{1}{1-\operatorname{Trace}(\boldsymbol{A}) / L^{2}},
$$

where $L$ is the ratio of fully stretched coil size to the random uncoiled size, i.e. $L^{2}=O(N)$. 
To produce the behaviour found in the simulations, we need to add an extra term to the stress which is proportional to the strain rate and which increases with the deformation according to $R^{4}(t) / N$. A suitable candidate with the correct tensorial shape is $2 \mu_{2}(\boldsymbol{A}: \boldsymbol{E}) \boldsymbol{A} / L$. This term gives the correct stress when the chain is fully stretched. This it is no longer necessary to make the elastic stress increase non-linearly. I therefore propose a new expression for the stress:

$$
\boldsymbol{\sigma}=-p \boldsymbol{I}+2 \mu \boldsymbol{E}+2 \mu_{2}(\boldsymbol{A}: \boldsymbol{E}) \boldsymbol{A} / \mathrm{L}+\mathrm{G} \boldsymbol{A} \text {. }
$$

The second microstructural equation should not be changed from the FENE form in order to limit the deformation to $L$.

There is considerable uncertainty in the form of the elastic term in the new proposed equation. Further research is needed to examine the elastic component of the stress when the chain is greatly extended. It is also necessary to examine the effect of hydrodynamic interactions between the beads of the chain. Some preliminary simulations with a poor aglorithm seemed to indicate that the stretching of the chain was reduced slightly from being affine (until the chain is fully stretched) and that the kinks scaling still applied.

\section{References}

[1] B. Debbaut, J.M. Marchal and M.J. Crochet, Numerical simulation of highly visco-elastic flows through an abrupt contraction. J. Non-Newtonian Fluid Mech., 29 (1988) 119-146.

[2] R.A. Keiller, Entry-flow calculations for the Oldroyd-B and FENE equations, J. Non-Newtonian Fluid Mech., 46 (1993) 143-178.

[3] D.V. Boger, M.J. Crochet and R.A. Keiller, On viscoelastic flows through abrupt contractions, J. Non-Newtonian Fluid Mech., 44 (1992) 267-280.

[4] J.M. Rallison and E.J. Hinch, Do we understand the physics in the constitutive relations, J. Non-Newtonian Fluid Mech., 29 (1988) 37-55.

[5] M.D. Chilcott and J.M. Rallison, Creeping flow of dilute polymer solutions past cylinders and spheres. J. Non-Newtonian Fluid Mech., 29 (1988) 381-432.

[6] U. Cartolos and J.M. Piau, Pressure drop scalings and structural stress contributions for complex flows of polymer solutions in thick solvents, J. Non-Newtonian Fluid Mech., 33 (1992) 55-83.

[7] A. Ambari, Thése de doctorat d'Etat, Université de Paris, 1986.

[8] D.F. James and J.H. Sarringer, Extensional flow of dilute polymer solutions, J. Fluid Mech., 97 (1980) 655-671.

[9] D.H. King and D.F. James, Analysis of the Rouse model in extensional flow. II. Stresses generated in sink flow by flexible macromolecules and by finitely extended macromolecules, J. Chem. Phys., 78 (1993) 4749-4754.

[10] G. Ryskin, Calculation of the effect of polymer additıve in a converging flow, J. Fluid Mech., 178 (1987) $423-440$.

[11] R.G. Larson, The unraveling of a polymer chain in a strong extensional flow, Rheol. Acta, 29 (1990) 371-384.

[12] P.S. Grassia, E.J. Hinch and L C. Nitsche, Computer simulation of Brownian motion of complex systems, J. Fluid Mech., (1994) in press.

[13] P.S. Grassia, Ph.D. thesis, Cambridge University, 1994.

[14] M. Moan and A. Magueur, personal communication, 1988

[15] V. Tirtaatmadja and T. Sridhar, A filament stretching device for measurement of extensional viscosity, J. Rheol., 37 (1993) 1081-1102. 